\title{
Editorial
}

\section{Constructions politiques : entre contingence et nécessité.}

Ce quatrième numéro d'Emulations est d'abord le premier du second volume, c'est-à-dire d'une nouvelle année qui débute. Après une première période réussie et empreinte d'éclectisme, la seconde année est celle des défis de plus grande ampleur: augmentation des propositions d'articles ainsi que de leur diversité géographique d'origine, travail sur la reconnaissance et la diffusion de la revue, publication et vente sur papier des volumes (articles des trois numéros de l'année), refonte du site internet, etc. Le premier défi que nous relevons est celui de la parution occasionnelle d'éditions thématiques. Il débute ici.

Ces pages constituent le premier tirage réellement thématique. Honneur au pouvoir: derrière une couverture étrange ou désagréable s'alignent une série d'articles mettant chacun en lumière une construction politique actuelle tout aussi étrange ou désagréable.

Le mot est là : il s'agit bien de « constructions » politiques. N'a-t-on pourtant pas déjà trop exploré la «construction sociale », n'en connaît-on pas par cœur les mécanismes ou l'importance de ses acteurs? En fait, plutôt que de continuer à alimenter l'interminable débat constructiviste de la science politique, nous voulons ici illustrer par des exemples forts les deux dimensions formant le cœur de tout fait politique. Les articles qui suivent sont autant de preuves qu'un fait politique est une construction, c'est-à-dire un événement mêlant contingence et nécessité dans un rapport défiant le principe de non-contradiction.

Le rapport entre contingence et nécessité constitue la tension fondatrice de tout événement politique compris comme construction. Occulter la dimension contingente, ce serait enfermer l'analyse dans un schéma causal simpliste niant un univers de possibles, ce serait affirmer la fatalité ou la naturalité de l'événement. Occulter la dimension de nécessité, ce serait laisser une part trop belle à l'utopie politique, ce serait sous-estimer l'ancrage de nos actions dans une rationalité dont il est difficile de s'écarter, ce serait enfin renier l'existence d'une réalité parfois brutale.

Tous nos articles reflètent de manière directe cette tension. D'abord, le récit de Steve Ringel illustre les contradictions qui structurent aujourd'hui l'aide humanitaire. Ses exemples tirés de son expérience de terrain n'illustrent que trop bien la force de la nécessité et la désespérante contingence qui semble parfois en résulter : tandis qu'il existe un nombre potentiellement illimité d'actions différentes, il a fallu que l'énergie se perde dans la mise en œuvre obligée d'une politique absurde dans un endroit où le temps prend pourtant une valeur vitale.

Ensuite, Xavier de Mûelenaere et Mathieu Roger cherchent à comprendre pourquoi l'Union Européenne n'a de cesse de présenter l'espace méditerranéen comme un tout cohérent, participant de ce fait à l'établissement d'une réalité unique et nécessaire. Mais croire que cette 
vérité tient de l'adéquation avec la réalité tient de la naïveté : cet espace euroméditerranéen si séduisant est une construction politique et stratégique dont la contingence n'est que trop aisément révélée par une étude en termes d'enjeux et de stratégies.

Mais l'origine de la contingence de nos actions est toujours plus profonde. Nadim Farhat ne montre pas autre chose en explorant les mécanismes de la mémoire collective lorsque celle-ci est confrontée à un événement grave, traumatique. Ce sont alors des mécanismes psychosociaux qui font qu'on ne pense que d'une seule manière à son passé, mais qui prouvent bien dans le même mouvement que d'autres façons de se souvenir sont possibles, par le biais d'un « travail de mémoire » développé à l'aune du travail de Paul Ricœur.

Enfin, le numéro se clôture par l'interview que m’a accordée le spécialiste allemand du philosophe Michel Foucault, Thomas Lemke. Qu'en retenir? Principalement que revenir à Foucault, ce serait se fournir les outils les plus pertinents pour comprendre les contours et les traits de cette rationalité à la fois contingente et nécessaire qui forme probablement le niveau d'analyse le plus pertinent pour saisir le sens des importantes mutations actuelles du politique. En discutant des notions de biopouvoir et de gouvernementalité, et en les illustrant par l'exemple des tests prédictifs génétiques, Lemke montre que face à la tension fondamentale de la construction politique, face à la contingence et à la nécessité, il est nécessaire de renouveler l'analyse politique.

Les constructions abordées sont donc "politiques » à double titre : en tant que lieux traversés par de multiples relations de pouvoir, mais aussi en tant qu'enjeux où une action autre, orientée vers une plus grande justice, est possible et demandée pour prendre la place de celles, opaques, qui les structurent lorsque le débat éthique est écarté. A la suite de nos contributeurs, je vous invite à aborder le politique comme une construction nécessaire et contingente, pour y développer une action responsable.

Stéphane Baele 